\section{Atividade física e qualidade de vida}

\author{
Physical activity and quality of life
}

Rodrigo Sinnott Silva ${ }^{1}$

Ivelissa da Silva ${ }^{1}$

Ricardo Azevedo da Silva ${ }^{2}$

Luciano Souza $^{1}$

Elaine Tomasi ${ }^{2}$

\footnotetext{
${ }^{1}$ Escola de Psicologia, Universidade Católica de Pelotas. RuaAlmirante Barroso 1202, Centro. 96010-280 Pelotas RS. rodrigo.ss.79@hotmail.com 2 Programa de Pósgraduação em Saúdee Comportamento, Escola de Psicologia, Universidade Católica de Pelotas.
}

Abstract Physical activity is believed to be a form to restore the health of the harmful effects caused by a stresfful work/study routine. The study comprises a transversal research which aims at analyzing the associations of the practice of sports activities in the quality of life of individuals. The samplewas comprised 107 professors, 111 employees and 638 students of the U niversidade Católica dePel otasU CPEL-RS, totalizing 863 participants. WHOQOL-brief/OM S wereused as a tool to evaluate quality of life as well as the Questionnaire of habitual physical activities translated and modified by N ahas - N uPAF/UFSC, 2001. Of the total, 394 are males and 469 are females. Of these, 313 aremoderately active, 210 inactive, 207 activeand 136 are very active. We can observe that, on average, the more active the person is, the better their quality of life is. M oreover, amongst the differencesin the quality of life of peoplewho practicephysical activities compared with those who do not practice any activity, are not only the aspects of physical health, but also psychological and cognitive aspects. These results serve as a motivation to professionals of different areas to encourage and support the definition of physical activities routine for their patients.

Key words Quality of life, Physical activity, Exercises
Resumo Acredita-se na atividade física como uma forma de restaurar a saúde dos efeitos nocivos que a rotina estressante do trabalho/ estudo traz. 0 estudo constitui uma pesquisa transversal que objetiva analisar as associações da prática de atividades esportivasna qualidade de vida dosindivíduos. A amostra foi constituída por 107 professores, 111 funcionários e638 estudantes da U niversidade Católica de Pel otas (U CPel), totalizando 863 participantes. Foram utilizados como instrumentos o W HOQOL-breve/OM S para avaliar a qualidade de vida e questionário de atividades físicas habituais traduzido e modificado por Nahas- N uPAF/UFSC, 2001. D o total, 394 são do sexo masculino e 469 do sexo feminino. Destes, 313 são moderadamente ativos, 210 inativos, 207 ativos e 136 são muito ativos. Pode-se observar que, em média, quanto mais ativa a pessoa é, melhor sua qualidade de vida. Além disso, dentre as diferenças na qualidade de vida das pessoas que praticam atividades físicas comparadascom asque não praticam, não estão apenasosaspectos de saúdefísica, mas também aspectos psicológicos e cognitivos. Estes resultados servem de estímulo a profi ssi onais de diferentes áreas para incentivarem e apoiarem a definição de rotinas de atividades físicas para seus pacientes.

Palavras-chave Qualidade devida, A tividadefísica, Exercícios 
Introdução

O esporte ea atividadefísica chegaram ao século XIX acompanhando as transformações políticas e sociais que começaram nos séculos anteriores, demonstrando, desde então, uma tendência a servir como uma tela de projeção da dinâmica social ${ }^{1}$. 0 exercício físico é uma forma de lazer e de restaurar a saúde dos efeitos nocivos que a rotina estressante do trabalho e do estudo traz.

O exercício, após superado o período inicial, éuma atividade usual mente agradável eque traz inúmeros benefícios ao praticante, quevão desde a melhora do perfil lipídico atéa mel hora da autoestima².

Qualidade de vida em saúde coloca sua centralidade na capacidade de viver sem doenças ou de superar as dificuldades dos estados ou condições de morbidade 3 .

Existem fatores físicos epsicológicosintervenientes na qualidade de vida das pessoas quando em situação de trabalho e que, dependendo do seu competentegerenciamento, proporcionarão condições favoráveis imprescindíveis ao melhor desempenho e produtividade4.

A inatividadefísica eum estilo de vida sedentário estão relacionados a fatores de risco para o desenvolvimento ou agravamento de certas condições médicas, tais como doença coronariana ou outras alterações cardiovasculares emetabólicas ${ }^{5}$.

Estudos realizados nos Estados Unidos afirmam que a prática sistemática do exercício físico está associada à ausência ou a poucos sintomas depressivos ou de ansiedade ${ }^{6}$.

Por outro lado, a exigência pela boa performance, o elevado grau de competitividade ea tolerância à frustração e ao estresse são algumas características que o ser humano encontra em seu dia-a-dia, segundo Rubio ${ }^{7}$.

Além dos benefícios, a atividade física também está associada a prejuízos para a saúdemental, aparecendo ligada a quadros como "exercício excessivo"8.

Pessoas que criam relações de dependência e compulsão pela prática de exercícios físicos passam a buscar nessas atividades mais a diminuição de sensações desagradáveis, como ansiedade, irritabilidade e depressão do que uma boa forma física?.

Portanto, este estudo visa analisar as relações entre atividades físicas e qualidade de vida em funcionários, professores e alunos da Universidade Católica de Pelotas (UCPEL).

\section{M etodologia}

Realizou-se uma pesquisa do tipo transversal em três segmentos da UCPEL - alunos, funcionários e professores.

A amostra foi determinada pelo método de Triola ${ }^{10}$, admitindo-se nível de confiança de $95 \%$ e proporção estimada de 0,5 para um total de 5.931 acadêmicose 760 professores efuncionários. Esta foi estratificada por segmento, obtendose 218 professores e funcionários com erro admissível de 5,6\% e 634 estudantes com erro admissível de 3,6\%.

Os estudantes foram amostrados de um total de 22 cursos da universidade, sorteando-se uma turma dos semestres iniciais, uma turma dos semestres intermediários e uma turma dos semestres finais. Todos os alunos das turmas sorteadas foram convidados a participar do estudo, obtendo-se, ao final, 645 estudantes; já os professores e funcionários foram selecionados de acordo com uma tabela de números al eatórios.

Os dados foram coletados através de um instrumento anônimo, auto- aplicável, com perguntas diretas e respostas precodificadas. N esse instrumento, para avaliação da qualidade de vida, foi utilizado o WHOQ OL-abreviado OM $\mathrm{S}^{11}$. Esse mensura, através de 26 questões, a qualidade de vida em quatro domínios - físico, psicológico, relações sociais e meio ambiente. 0 domínio físico avalia dor e desconforto, energia e fadiga, sono e repouso; o domínio psicológico, os sentimentos positivos, pensar, aprender, memória e concentração, auto-estima, imagem corporal (aparência), sentimentos negativos; o domínio relações sociais questiona as relações pessoais, suporte (apoio) social e atividade sexual; enquanto o domínio meio ambiente, a segurança física e proteção, ambiente no lar, recursos financeiros, cuidados de saúde e sociais, oportunidade de adquirir informações e habilidades, oportunidades de recreação e lazer, ambiente físico e locomoção.

$\mathrm{Na}$ avaliação das atividades físicas, foi utilizado o questionário de atividades físicas habituais, desenvolvido originalmente por Pate; subdivide-se em atividades ocupacionais diárias e atividades de lazer através de onze questões, que ao final classifica em inativo, moderadamente ativo, ativo e muito ativo. A referida escal a foi traduzida e modificada por $\mathrm{N}$ ahas ${ }^{12}$. Além disso, os entrevistados respondiam questões referentes a dados pessoais como idade, sexo, profissão, estado civil eturno predominante de trabalho/aula.

Os professores e funcionários foram avaliados nas unidades em que se encontram lotados, 
enquanto os estudantes, em suas respectivas salas de aula.

Os dados foram tabulados e analisados através do software SPSS versão 10.0 para Windows ${ }^{13}$. Foram obtidas frequências simples das variáveis e os cruzamentos foram avaliados através de regressão linear em análise bivariada e controlados através de um modelo hierárquico em que as variáveis com um valor $p \leq 0,20$ no teste de razões de verossimilhança permaneciam no modelo.

A pesquisa foi autorizada pela Universidade Católica de Pelotas e solicitou-se o consentimento informado de cada um dos participantes, tendo esses autonomia para se recusar a participar.

\section{Resultados}

A amostra foi composta por 863 participantes, sendo 638 estudantes (aumentando ainda mais a confiabilidade dos resultados), 111 funcionários e 107 professores ( $a$ amostra real valida o erro e

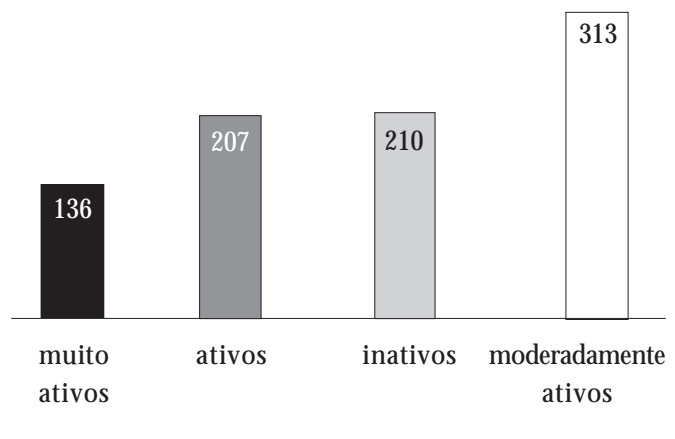

Gráfico 1. Padrão de atividade física. confiabilidade dos resultados). Destes, 394 são do sexo masculino e469, do sexo feminino. Quanto ao estado civil, 594 são solteiros, 173, casados, 50 , divorciados, 10 viúvos e 36 marcaram a opção outros. Com relação ao turno predominante de trabalho/estudo, 337 o fazem à noite, 54, à tarde, 49, pela manhã, 295, manhã e tarde, 65 , tarde e noite, 12 , manhã e noite e 29 , manhã, tarde enoite.

Das 863 pessoas que responderam o questionário, 313 são moderadamente ativas, 210 são inativas, 207 são ativas e 136 são muito ativas (Gráfico 1).

$\mathrm{Na}$ análise bivariada (Tabela 1), relacionando cada domínio do WHOQOL com a variável padrão de atividade física, pessoas muito ativas apresentaram significativamente maiores escores de qualidade de vida em relação aos inativos, exceto quanto ao domínio relações sociais; no entanto, a variação neste domínio não foi significativa ( $B=0,51$ IC $95 \%-0,57$ a 1,58 ).

Quando comparada a variável sexo com o domínio ambiente físico, foi observada uma diferença significativa, indicando que o sexo feminino tem melhor qualidade de vida neste domínio que o sexo masculino ( $B=-1,80$ IC $95 \%-3,26$ a -0,25). Os cruzamentos com o domínio ambientepsicológico ecom o domínio meio ambiente apontam na mesma direção; no entanto, em ambos não houve significância. No cruzamento da variável sexo com o domínio relações sociais, a situação foi inversa, apontando melhor qualidade de vida no sexo masculino; contudo, foi uma diferença sem significância estatística $(B=$ 0,43 IC 95\% - 1,75 a 2,60).

A variável idade, quando comparada ao domínio ambiente físico, ambiente psicológico e meio ambientemostrou quequanto maior a idade dos participantes, maior os escores de qualidade de vida; porém, somente no cruzamento

Tabela 1. Cruzamentos em análise bivariada.

\begin{tabular}{|c|c|c|c|c|c|c|c|c|}
\hline \multirow[b]{2}{*}{$\begin{array}{c}\text { Variável } \\
\text { independente }\end{array}$} & \multicolumn{2}{|c|}{$\begin{array}{l}\text { Domínio físico } \\
\text { bruto }\end{array}$} & \multicolumn{2}{|c|}{$\begin{array}{c}\text { Domínio psicológico } \\
\text { bruto }\end{array}$} & \multicolumn{2}{|c|}{$\begin{array}{l}\text { Domínio relações } \\
\text { sociais bruto }\end{array}$} & \multicolumn{2}{|c|}{$\begin{array}{l}\text { Domínio meio } \\
\text { ambiente bruto }\end{array}$} \\
\hline & B (IC95\%) & $\mathrm{p}$ & B (IC95\%) & $\mathrm{p}$ & B (IC95\%) & $P$ & B (IC95\%) & $\mathrm{p}$ \\
\hline Sexo & $-1,80(-3,26$ a $-0,35)$ & 0,015 & $-1,10(-2,49$ a 0,29$)$ & 0,122 & $0,43(-1,75 A \quad 2,60)$ & 0,700 & $-1,14(-2,97$ a 0,70$)$ & 0,224 \\
\hline Profissão & $-2,24(-3,26$ a $-1,22)$ & 0,001 & $-0,54(-1,52$ a 0,44$)$ & 0,282 & $1,12(-0,41$ a 2,66$)$ & 0,151 & $0,95(-0,35$ a 2,24$)$ & 0,153 \\
\hline Idade & $3,19(2,13$ a 4,25$)$ & 0,001 & $0,46(-0,57$ a 1,50$)$ & 0,380 & $-1,61(-3,22$ a 0,00$)$ & 0,050 & $0,43(-0,93$ a 1,79$)$ & 0,535 \\
\hline $\begin{array}{l}\text { Padrão de } \\
\text { atividade física }\end{array}$ & $1,39(0,68$ a 2,11$)$ & 0,001 & $1,60(0,92$ a 2,28$)$ & 0,000 & $0,51(-0,57$ a 1,58$)$ & 0,354 & $1,72(0,83$ a 2,61$)$ & 0,000 \\
\hline
\end{tabular}


com 0 ambiente físico as diferenças se mostram significativas ( $B=3,19$ I C $95 \% 2,13$ a 4,25). Uma diferentesituação foi encontrada no cruzamento com o domínio relações sociais, em que, com diferenças signicativas, quanto menor a idade do participante, maior seu escore em qualidade de vida ( $B=-1,61$ IC $95 \%-3,22$ a 0,00).

Quando analisamos a variável profissão, obteve-se significância no domínio ambientefísico, apontando que funcionários têm maiores escores de qualidade de vida do que professores e, estes, maiores queos dosalunos ( $B=-2,24 \mid C 95 \%$ $-3,26$ a -1,22). Nos demais domínios, não houve significância entre as diferenças; no cruzamento com o domínio ambiente psicológico, os escores apontam melhor qualidade de vida nos funcionários e pior nos alunos, contrário aos achadosnos domínios relações sociais e meio ambiente.

Em uma análise multivariada (Tabela 2), de acordo com o modelo hierárquico estabelecido (domínio ambiente físico: nível 1: sexo, nível 2: profissão, nível 3: idade, nível 4: atividade física/ domínio ambientepsiológico: nível 1: sexo, nível 2: atividadefísica/domínio ambienterelações sociais: nível 1: sexo, nível 2: profissão, nível 3: idade, nível 4: atividade física/domínio meio ambiente: nível 1: profissão, nível 2: atividade física), controlando a variável sexo ao analisar o cruzamento da variável profissão com os quatro domínios do WHOQOL, percebeu-se variação ape nas no domínio ambiente físico, que apresentou maior significância na diferença entre os valores, confirmando o resultado obtido anteriormente. $(B=-2,17$ IC 95\% - 3,19 a-1,15)

Analisando a variável idade, controlando as variáveis sexo e profissão, obteve- se perda da significância no domínio ambiente físico ( $B=2,75$ IC 95\% 1,45 a 4,05) euma tendência a significância no domínio relações sociais ( $B=-0,10$ IC $95 \%$ $-0,22$ a 0,14$)$. Os demais domínios se mantive ram sem alterações.
Controlando as variáveis sexo, profissão e idadeno cruzamento da variável padrão de atividade física com os domínios do WHOQOL, obteve-se um aumento da significância do domínio ambiente físico ( $B=1,59$ IC $95 \%$ 0,87 a 2,31) e uma equivalência de resultados semelhantes à análise bivariada apresentada anteriormente.

\section{Discussão}

Ao contrário de estudos como os de Alves et al. ${ }^{14}$, que apontam para uma elevada prevalência de inatividadeem populaçõessimilares, os dados encontrados no presente estudos mostram maior prevalência de pessoas ativas em geral, dados que apóiam um estudo futuro que possa confirmar uma melhora na qualidade de vida em decorrência do abandono do estilo de vida sedentário.

Resultados que mostram pior qualidade de vida em estudantes são preocupantes, visto que ficou comprovada uma correlação significativa entre atividade física e qualidade de vida e adolescentes sedentários têm grande probabilidade de se tornarem adultos sedentários, como foi referido em Alves et al. ${ }^{14}$, justificando a adoção de rotinas de exercícios físicos em escolas, universidades e a devida indicação de profissionais da saúde.

Associações que indicam melhora em estados físicos e psi cológicos também são encontradas em Assumpção et al. ${ }^{15}$ quando os comparam à prática de atividades físicas.

Em concordância com estudos realizados por Pereira et al. ${ }^{16}$, dentre os quatro domínios, o que mais explicou a qualidade de vida global foi 0 físico.

Os resultados em relação à qualidade de vida eà prática de atividade física não se modificaram quando controladas as variáveis sexo, idade e profissão, contariando o que relatam M elanson

Tabela 2. Cruzamentos ajustados em análise multivariada.

\begin{tabular}{|c|c|c|c|c|c|c|c|c|}
\hline \multirow[b]{2}{*}{$\begin{array}{c}\text { Variável } \\
\text { independente }\end{array}$} & \multicolumn{2}{|c|}{$\begin{array}{l}\text { Domínio físico } \\
\text { ajustado }\end{array}$} & \multicolumn{2}{|c|}{$\begin{array}{c}\text { Domínio psicológico } \\
\text { ajustado }\end{array}$} & \multicolumn{2}{|c|}{$\begin{array}{l}\text { Domínio relações } \\
\text { sociais ajustado }\end{array}$} & \multicolumn{2}{|c|}{$\begin{array}{c}\text { Domínio meio } \\
\text { ambiente ajustado }\end{array}$} \\
\hline & B (IC95\%) & $p$ & B (IC95\%) & $p$ & B (IC95\%) & $P$ & B (IC 95\%) & $p$ \\
\hline Sexo & - & - & - & - & - & - & - & - \\
\hline Profissão & $-2,17(-3,19$ a $-1,15)$ & 0,000 & - & - & - & - & - & - \\
\hline Idade & $2,75(1,45$ a 4,05$)$ & 0,171 & - & - & $-0,10(-0,22$ a 0,14$)$ & 0,083 & - & - \\
\hline $\begin{array}{l}\text { Padrão de } \\
\text { atividade física }\end{array}$ & $1,59(0,87$ a 2,31$)$ & 0,000 & $1,59(0,89$ a 2,28$)$ & 0,000 & $0,27(-0,81$ a 1,35$)$ & 0,622 & $1,67(0,77$ a 2,57$)$ & 0,000 \\
\hline
\end{tabular}


et al. ${ }^{17}$, que as informações relacionadas à prática habitual de atividadefísica encontradas mediante a aplicação de questionários podem diferir por conta da natureza e das especificações das questões apresentadas, o que deverá variar de acordo com o sexo, a idade, o desenvolvimento cognitivo e o contexto sociocultural em que os sujeitos estão inseridos.

Apesar denão apontar uma variação significativa no cruzamento entre padrão de atividadefísica e o domínio relações sociais, as maiores médias estão em pessoas ativas em geral, e a menor, em pessoas inativas. Dados que merecem atenção quando se pensa no aumento das atividades em grupo e das novas interações pessoais proporcionadas pelas atividades físicas, de modo que esta torna-se uma oportunidade de "investimento social", como também é referenciado em Laberg ${ }^{18}$.

Independente desexo, idade eprofissão, ficou evidenciado quea atividade física acarreta mel horas na qualidade de vida em todos os aspectos.

Os resultados não fornecem dados para especular sobre as razões das indicações de melhor qualidade de vida encontrada nos funcionários, pois partilhamos das idéias de Sato, citado por Lacaz ${ }^{19}$, de que a qualidade de vida também esta ligada ao mundo subjetivo (desejos, vivências, sentimentos), aos valores, crenças, ideologias e aos interesses econômicos e políticos. Porém, estas questões devem ser estudadas tendo em vista os fatores de influência na qualidade de vida em situação de trabalho.

Não analisado em nosso estudo, recomendamos atenção ao desenvolvimento da dependência ao exercício ${ }^{9}$ ea prática excessi va ${ }^{8}$ do mesmo relacionadosà qualidadedevida. Assim como mostram estudos recentes ${ }^{20}$, compartilhamos da idéia de que o exercício éuma intervenção de baixo custo, que pode promover saúde em vários aspectos quando conscientemente realizado.

\section{Colaboradores}

RS Silva participou da concepção, análise dos dados e redação final. I Silva participou da pesquisa e metodologia. RA Silva foi revisor crítico e orientador. L Souza foi revisor crítico e participou da análise dos dados e E Tomasi participou da análise dos dados.

\section{Referências}

1. Rubio K. M edalhistas olímpicos brasileiros: memórias, histórias e imaginário. São Paulo: Casa do Psicólogo/FAPESP; 2006.

2. Tofler GH, Mittleman MA, Muller JE. Physical activity and the triggering of myocardial infarction: the case for regular exercise. Heart 1996; 75:323-325.

3. M inayo M CS, Hartz ZM A, Buss PM. Quality of life and health: a necessary debate. Cien Saude Colet [periódico na Internet]. 2000 [acessado 2006 nov 21];5(1):[cerca de 12 p.]. Disponível em: http://www. scielo.br/scielo.php?script=sci_arttext\& pid=S1413$81232000000100002 \& \operatorname{lng}=e n \& n r m=i s 0$

4. Fernandes EC. Qualidade de vida no trabalho: como medir para melhorar. Salvador: Casa da Qualidade; 1996.

5. Maron BJ. The paradox of exercise. N Engl J Med 2000; 343(19):1409-1411.

6. Boscolo RA, Esteves AM, Mello MT, Tufik S. O exrcício físico e os aspectos psicobiológicos. Rev Bras M ed Esporte 2005; 11(3):203-207.

7. Rubio K, organizadora. Psicologia do esporte: interfaces, pesquisa e intervenção. São Paulo: Casa do Psicólogo; 2000.
8. Peluso M AM , Andrade LHSG. Atividade física e saúde mental: a associação entre exercício e humor. Clinics [periódico na Internet]. 2005 [acessado 2006 nov 21]; 60(1):[cerca de 10 p.]. Disponível em: http:/ /www.scielo.br/scielo.php?script=sci_arttext \& pid=S1807-59322005000100012\&lng=pt\&nrm=iso

9. Bicudo F. Praticar exercícios é terapia para dependentes de atividades físicas. Jorn da paulis. [periódico na Internet]. 2001 [acessado 2006 jul 03];(157). Disponível em: http://tuiuiu.epm.br/comunicacao/ jpta/ed157/pesq5.htm

10. Triola MF. Introdução à estatística. 9ạ ed. Rio de Janeiro: LTC; 2005.

11. Fleck MPA, organizador. OMS. Divisão de Saúde M ental. [base de dados na Internet] 1998 [acessado 2004 nov 16]. Disponível em: www.ufrgs.br/psiq/ whogol.html

12. Nahas M V. Atividade física, saúde e qualidade de vida: Conceitos e sugestões para um estilo de vida ativo. Londrina: M iografi; 2001.

13. SPSS for Windows, Rel. 10.0.0 [programa de computador]. Chicago: SPSS Inc.; 1999. 
14. Alves JGB, Alves RV, Montenegro FMU, Oliveira FA. Prática de esportes durante a adolescência e atividade de lazer na vida adulta. Rev Bras Med Esporte 2005; 11(5):291-294.

15. Assumpção LOT, Morais PP, Fontoura H. Relação entre atividade física, saúde e qualidade de vida. Notas Introdutórias. EF y Desp. [periódico na internet] 2002 [acessado 2006 dez 04]; 52: [cerca de 3 p.] Disponível em: http://www.efdeportes.com/ efd52/saude.htm

16. Pereira RJ, Cotta RM M, Franceschini SCC, Ribeiro $\mathrm{RCL}$, Sampaio RF, Priore SE, Cecon PR. Contribuição dos domínios físico, social, psicológico e ambiental para a qualidade de vida global de idosos. Rev Psiquiatr RS [periódico na Internet]. 2006 [acessado 2006 nov 21]; 28(1):[cerca de 12 p.]. Disponível em: http://www.scielo.br/scielo.php? script $=$ sci_arttext $\&$ pid $=$ S0101-81082006000100005 $\& \operatorname{lng}=\mathrm{pt} \& \mathrm{nrm}=\mathrm{iso}$

17. Melanson EL, Freedson PS. Physical activity assessment: a review of methods. Crit Rev Food Sci Nutr 1996; 36:385-396.
18. Laberge S. Toward an integration of gender into Bourdieu's concept of cultural capital. Sociol of Spor Journ. 1995; 12:132-146.

19. Lacaz FAC. Quality working life and health/illness. Cien Saude Colet [periódico na Internet]. 2000 [acessado 2006 nov 21];5(1):[cerca de 11 p.] Disponível em: http://www.scielo.br/scielo.php?script=sci 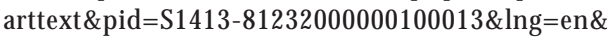 $\mathrm{nrm}=\mathrm{iso}$

20. Valim V. Benefícios dos exercícios físicos na fibromialgia. Rev. Bras. Reumatol. [periódico na Internet]. 2006 [acessado 2006 nov 21];46(1):[ cerca de 7 p.]. Disponível em: http://www.scielo.br/scielo. php?script $=$ sci arttext $\&$ pid $=$ S0482-50042006000 $100010 \&$ Ing $=$ pt $\&$ nrm $=$ iso

Artigo apresentado em 18/12/2006

Aprovado em 29/06/2007

Versão final apresentada em 27/07/2007 